\title{
Static Experiments Research on Multi-Layer Steel Plate Isolator
}

$$
\text { Yan Hui, a , Zhang Lu }{ }^{2, b} \text {, Dai Guanghao }{ }^{3, \mathrm{c}} \text { and Jiang Hongyuan }{ }^{4, \mathrm{~d}}
$$

${ }^{1,2,4}$ Harbin Institute of Technology, 92, Xi Da Zhi, Harbin, P. R. China, 150001

${ }^{3}$ The 703 Research Institute of China Shipbuilding Industry Corporation,452, Hong Qi, Harbin, P. R.

China,150030

ayanhui@hit.edu.cn, ${ }^{\mathrm{b}}$ zhanglu916hit@163.com, ${ }^{\mathrm{c}}$ sdcxlikai@126.com,

djhy_hit@hit.edu.cn(Corresponding author)

Keywords: corrugated steel plate isolator, stiffness, energy dissipation coefficient, hysteresis loop

\begin{abstract}
This paper developed multi-layer corrugated steel plate isolators in order to meet the needs of isolation in marine power systems, and the main parameters which affecting the performance of corrugated steel plate isolator are studied through the static experiments. The experimental results show that multi-layer corrugated steel plate isolator obtains good hysteresis damping characteristics. The stiffness and energy dissipation coefficient of isolator both have nonlinear relationship with the deformation amplitude. The performance of isolator depends mainly on the thickness of plate and the corrugated type and other parameters. The experimental results are helpful for the application of multi-layer corrugated steel isolator in marine power systems.
\end{abstract}

\section{Introduction}

With the development of marine technology, the vibration created by hull has gradually become one of the prime failure reasons of the ship's parts[1]. Vibration control technology is particularly urgent in marine power systems, and people have been committing to take various measures to reduce or isolate vibration. Due to marine power systems' special working conditions and environment, one of the effective ways to solve the vibration problem is to use the new isolator with dry friction damping characteristics[2]. Recent studies mostly focus on the new metal isolator with the performance of dry friction damping, such as metal rubber isolator, wire rope isolator and metal wire mesh isolator, some of them have been successfully applied in aerospace fields[3-6]. The multi-layer corrugated steel isolator has the advantages of simple structure, better processing technology, preferable environment adaptability and the significant damping effect, so it has broad application prospects[7].

This paper developed multi-layer corrugated steel plate isolators to solve the vibration problem of marine power systems which are working in harsh environment conditions. The stiffness and damping characteristics of isolators with different structural parameters are analyzed based on the data from static experiments.

\section{Structural design of multi-layer corrugated steel isolator}

Table 1 Structure parameter table of the multi-layer steel plate isolator

\begin{tabular}{cccc}
\hline wave type & $\begin{array}{c}\text { thickness } \delta \\
(\mathrm{mm})\end{array}$ & $\begin{array}{c}\text { height } / h \\
(\mathrm{~mm})\end{array}$ & layers $/ n$ \\
\hline \multirow{2}{*}{ three wave } & 0.52 & 4 & 25 \\
& 0.28 & & 46 \\
one wave & 0.52 & 4 & 25 \\
& 0.28 & & 46 \\
\hline
\end{tabular}

Vibration isolator is assembled by steel plates and shell, in this paper, two types of corrugated 
steel plate are primarily designed, i.e. one wave type and three wave type. Each type of corrugated steel plate has two sizes of thickness. Ensuring the overall height of isolator is $17 \mathrm{~mm}$, the number of steel plate layers are selected according to the thickness of steel plate and the height of corrugated plate. Structural parameters of multi-layer plate isolator are shown in Table 2.

Laminated steel plates with same parameters are put into the isolator shell, covered by upper cover and fixed by screw. Vibration isolator mainly bear one-way axial load, we can get different performance of isolator by change corrugated type or thickness of steel plate. The structural diagram of steel plate and isolator are shown in Fig. 1.

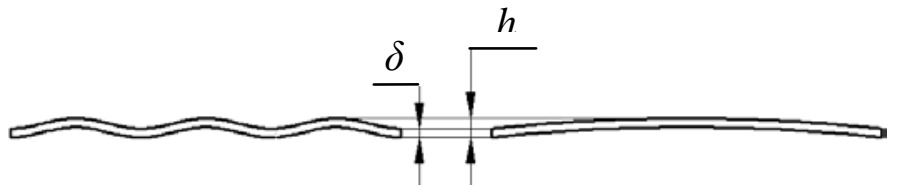

three wave

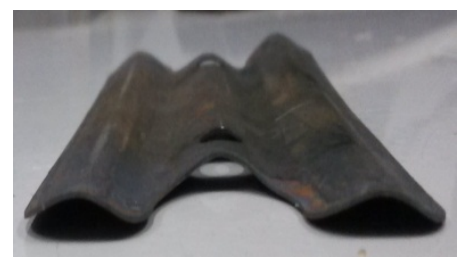

(a) Different types of steel plate
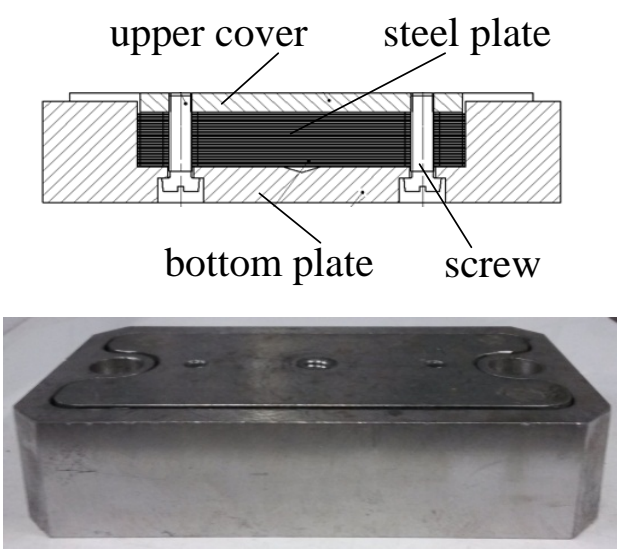

(b) Structure of multi-layer steel isolator

Fig.1 Structural diagram of steel plate and isolator

\section{Analysis of the static experiments of multi-layer corrugated steel isolator}

The hysteresis loop of the multi-layer steel plate isolator with different structural parameters and different deformation amplitudes was presented through the static experiments. Energy dissipation coefficient and stiffness of isolator which are used to analyze damping characteristics and stiffness of multi-layer steel isolator are calculated according to the formula.

Energy dissipation coefficient $\Psi$ is used to represent the damping characteristics of dry friction damping system [8]:

$$
\psi=\Delta W / W
$$

In Eq.1, $\Delta W$ represents the dissipation of energy in the form of dry friction in a deformation period, it can be calculated by the area enclosed of hysteresis loop; $W$ represents maximum potential energy of deformation of dry friction system. Get the centerline of the loading and unloading curve, $W$ can be calculated by the area enclosed between the center line and coordinate axis.

Hysteresis loop of multi-layer corrugated steel isolator. Vibration isolator's stiffness and damping characteristics are analyzed by the hysteresis loop which is drawn by the data of static experiments of multi-layer steel plate isolator with different parameters.

Fig. 2 shows the hysteresis loop of isolators with four different structural parameters under different deformations of $0.1 \mathrm{~mm}, 0.2 \mathrm{~mm}, 0.3 \mathrm{~mm}, 0.5 \mathrm{~mm}, 0.7 \mathrm{~mm}$ and $1 \mathrm{~mm}$. It can be seen from the Fig.2, vibration isolator has stronger nonlinear stiffness; and when the deformation increases the hysteresis loop enclosed area will increase, thereby the damping properties of isolators are better due to the increased energy dissipation. 


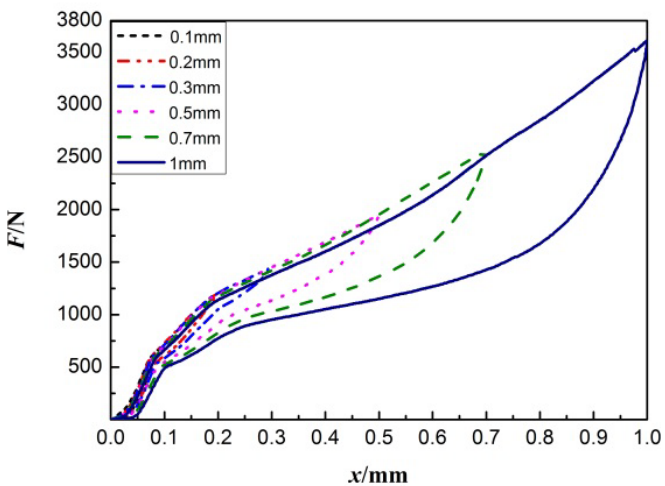

(a) 1wave , $\delta=0.28 \mathrm{~mm}$

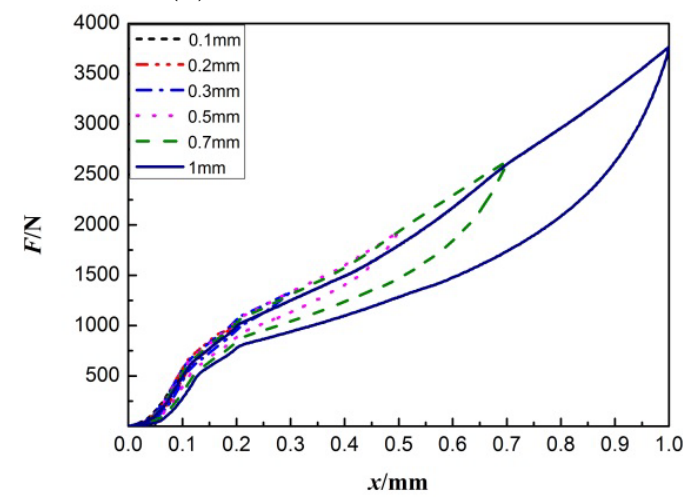

(c) 3wave , $\delta=0.28 \mathrm{~mm}$

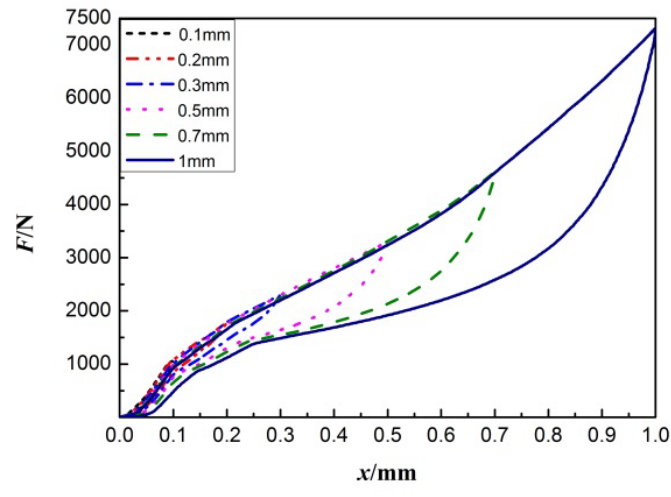

(b) 1wave , $\delta=0.52 \mathrm{~mm}$

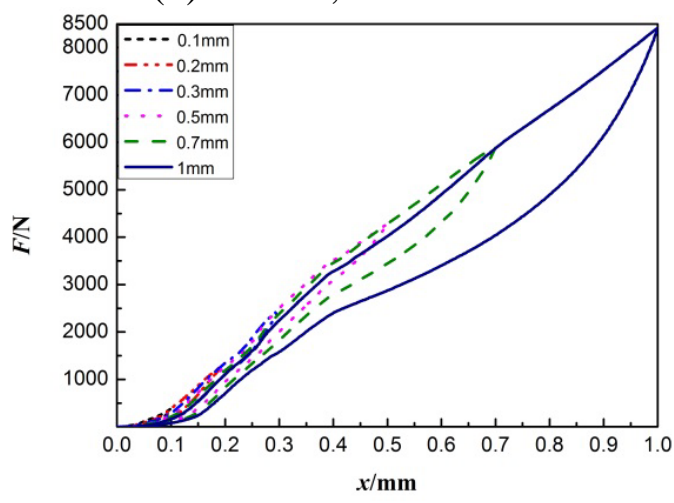

(d) 3wave , $\delta=0.52 \mathrm{~mm}$

Fig.2 Hysteresis loop of isolators with different structures under different deformation

Stiffness of multi-layer corrugated steel isolator. Fig.3 shows the stiffness curve of isolators with different corrugated type and different thickness of steel plate, where $\mathrm{A}$ is the amplitude of the deformation, $\mathrm{K}$ is the stiffness.

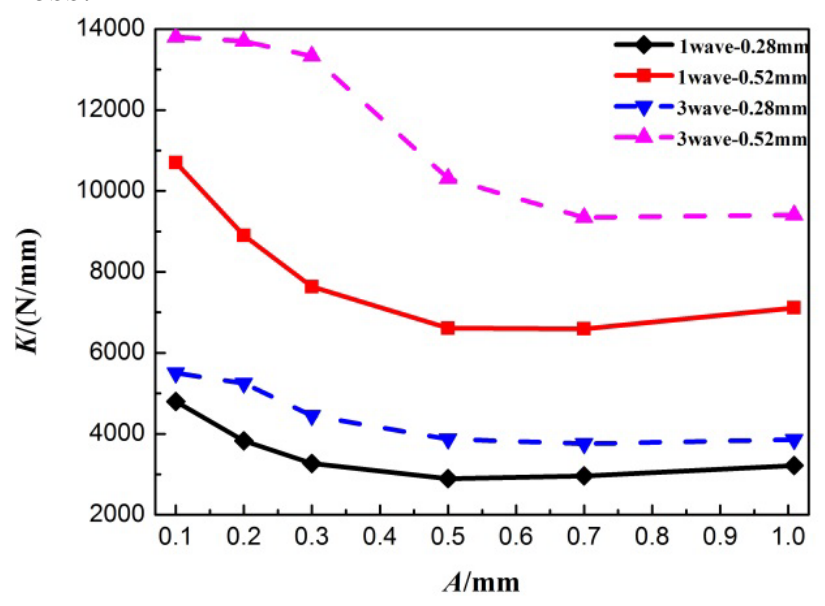

Fig.3 Stiffness curve of isolator with different structural parameters

It can be seen the stiffness curve of isolators with different structural parameters are all nonlinearly. The stiffness of the isolators change sharply in a downward trend before the turning point, then increase slowly. It means the optimum operating range of the isolator is after the turning point, because in this range, the resonance frequency of vibration isolator keeps essentially unchanged. And to isolators with the same corrugated steel plate, its stiffness will increase along with the increase of the thickness of steel plate, because vibration isolator loads more as the thickness of steel plate raises; furthermore when the thickness of steel plate is the same, compared with one wave steel plate isolator, the stiffness of three wave steel plate isolator is always greater, because when the quantity of waves increases, the contact area between steel plates raise, and 
vibration isolator loads more too, which result in bigger stiffness of isolators.

Energy dissipation coefficient of multi-layer corrugated steel isolators. Fig.4 indicates the energy dissipation coefficient curve of isolators with different corrugated type and different thickness of steel plate, where $A$ is the amplitude of deformation, $\psi$ is the energy dissipation coefficient.

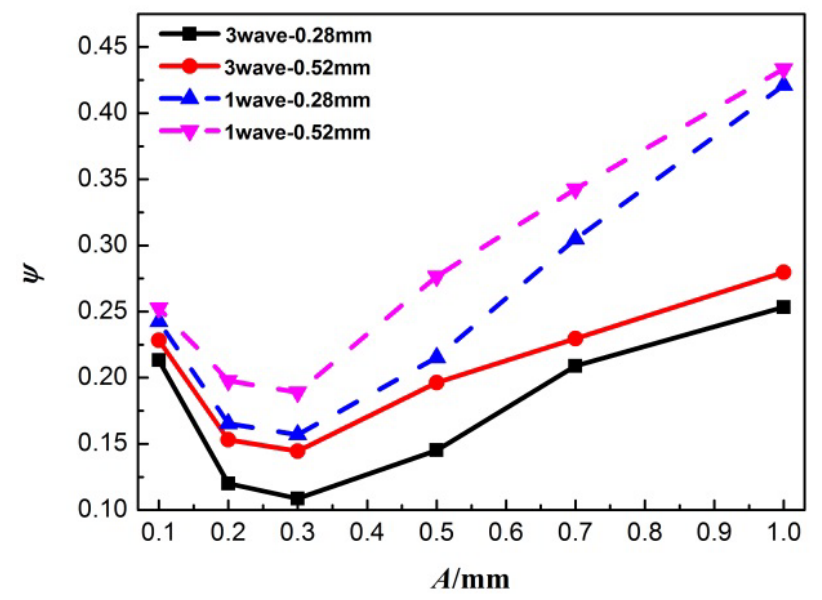

Fig.4 Energy dissipation coefficient of isolators with different structural parameters

It can be seen that the energy dissipation coefficient of isolators decreases and then increases along with the increase of deformation. The thicker the steel plate is, the larger the isolator's energy dissipation coefficient will be, because vibration isolator bears more load when the thickness of steel plate raises, and larger tangential load which is located in the contact area between plates will be created; then more energy dissipation during a deformation period can be obtained and according to the Eq.1, greater energy dissipation coefficient will be gotten. This demonstrates the thicker the steel plate is, the better the damping effect of isolator will be.

Furthermore when the thickness of steel plate is the same, compared with three wave steel plate isolator, energy dissipation coefficient of one wave steel plate isolator is always greater. The main reason is that under same deformation, maximum potential energy of deformation of isolators with three wave steel plate is larger due to its bigger stiffness, and the slip distance between layers of three wave steel plate isolator is less than that of one wave steel plate isolator. According to the Eq.1, energy dissipation coefficient of three wave steel plate isolators will be less.

\section{Conclusions}

Damping vibration isolation is achieved by the dry friction effect between the layers of steel plate in multi-layer corrugated steel plate isolator. In this paper, the hysteresis loop of multi-layer steel plate isolators are drawn through the static experiments, then the stiffness and energy dissipation coefficient curve under different deformation is obtained. Studies show that the stiffness and energy dissipation coefficient of multi-layer steel plate isolator is nonlinearly along with the increase of deformation; isolator's stiffness and damping characteristics are influenced by the thickness of steel plate and the corrugated type. For the steel plate of same corrugated type, the thicker the steel plate is, the greater the isolator's stiffness and energy dissipation coefficient will be; for the same thickness of steel plate, energy dissipation coefficient of one wave steel plate isolator is larger, however, stiffness of three wave steel plate isolator is larger. The adjustment of vibration isolation performance can be achieved to make it meet the requirement of different working conditions by changing the structural parameters of multi-layer corrugated steel plate isolator. 


\section{References}

[1] Yang Benle: Doctoral dissertation of DalianMaritimeUniversity, Research on the Vibration Characteristics of Marine Floating Raft Vibration Isolation System(2011)

[2] Oden J T, Martins J A C: Computer Methods in Applied Mechanics and Engineering Vol.52 (1985), p. 527-634

[3] Jones D I G: Journal of Sound and Vibration,Vol.35(1974), p. 417-27

[4] JIANG Hong-yuan, Ao Hong- rui, XIA Yu-hong , et al: Journal of Machine Design, Vol.11 (2002), p. 11-14

[5] Luo Yi: Doctoral dissertation of Chongqing University,Design of New Type Steel Wire Isolator and The Study of Its Characteristics (2012)

[6] ZHAO Jun-sheng, FAN Wen-x in, ZHANG Bao-cheng: Journal of North China Institute of Technology, Vol.01(2004), p. 28-30.

[7] Пономарев Ю, Проничев Ю, Чегодаев Д:Изд-во Самарского государственного аэрокос-мического университета, 1998,

[8] JIANG Hongyuan, WANG Yu , AO Hongrui , YAN Hui:Journal of Experimental Mechanics, Vol.04(2004), p. 423-429 